\title{
An Experimental Evaluation of Position Estimation Methods for Person Localization in Wireless Sensor Networks
}

\author{
Johannes Schmid ${ }^{1}$, Frederik Beutler ${ }^{2}$, Benjamin Noack ${ }^{2}$, Uwe D. Hanebeck ${ }^{2}$, \\ and Klaus D. Müller-Glaser ${ }^{1}$ \\ 1 Institute for Information Processing Technology (ITIV), www.itiv.kit.edu, \\ 2 Intelligent Sensor-Actuator-Systems Laboratory (ISAS), \\ Institute for Anthropomatics, www.isas.uka.de, \\ Karlsruhe Institute of Technology (KIT), Germany
}

\begin{abstract}
In this paper, the localization of persons by means of a Wireless Sensor Network (WSN) is considered. Persons carry on-body sensor nodes and move within a WSN. The location of each person is calculated on this node and communicated through the network to a central data sink for visualization. Applications of such a system could be found in mass casualty events, firefighter scenarios, hospitals or retirement homes for example.

For the location estimation on the sensor node, three derivatives of the Kalman filter and a closed-form solution (CFS) are applied, compared, and evaluated in a real-world scenario. A prototype 65-node ZigBee WSN is implemented and data are collected in in- and outdoor environments with differently positioned on-body nodes. The described estimators are then evaluated off-line on the experimentally collected data.

The goal of this paper is to present a comprehensive real-world evaluation of methods for person localization in a WSN based on received signal strength (RSS) range measurements. It is concluded that person localization in in- and outdoor environments is possible under the considered conditions with the considered filters. The compared methods allow for sufficiently accurate localization results and are robust against inaccurate range measurements.
\end{abstract}

Keywords: Sensor Networks, Person Localization, Signal Strength, Location Estimation, Kalman Filter

\section{Introduction}

With recent and upcoming location-based services, personal localization systems (PLS) become a more and more important issue. On one hand, end-users can be provided with their own current position, on the other, this information can be communicated to a central authority. In outdoor environments, this localization can be achieved cheap and efficiently with satellite based positioning techniques. However, especially indoor environments but also places where valid satellite 
signals are not available at all times, are yet an open challenge. To provide this localization, there are already expensive and accurate real time location systems (RTLS) based on various technologies such as the upcoming ultra-wideband (UWB) technology [25], infrared light signals in iGPS systems [4] or others. Additionally, there are approaches to node tracking from the WSN community, where localization has been in the focus of research for more than ten years now [2].

Alternatively, there have also been major improvements in inertial navigation systems recently. These pedestrian dead reckoning (PDR) concepts, make use of inertial sensor measurements for position estimation [8].

In this paper, the localization of moving persons in a WSN via RSS range measurements is considered. The WSN enables the communication of the calculated position estimation to a central data sink. The proposed system allows for ad-hoc deployment and cheap implementation. Potential application areas include the tracking of patients and doctors in a hospital or in the compounds of a retirement home, the navigation of visitors in a museum or also the logistical coordination of a firefighter/police operation in and around a burning building.

Stochastic information filters [5] are used to cope with the RSS inherent high measurement fluctuations. The goal of this paper is to evaluate the performance of efficiently computable Bayesian state estimation for the localization of moving persons by means of a WSN. For this purpose, three derivatives of the Kalman filter, the Extended Kalman filter (EKF, [10]), the Unscented Kalman filter (UKF, [9]) and the recently developed Analytic Moment Calculation (AMC, [3]) filter are compared with a closed form solution (CFS, [7,22]). This comparison is carried out off-line on a dataset from a 65 -node WSN experiment. It is shown that mobility and high measurement rates allow for a reasonable localization accuracy.

The remainder of this paper is organized as follows. After a short survey on the related work (Section 2), a system model is established and the filters are described (Section 4). The gathered experimental data is presented in Section 5 and the evaluated localization methods are compared in Section 6. In Section 7 the paper is concluded and the next development steps are briefly outlined.

\section{State of the Art}

Localization in wireless sensor networks (WSN) has been a topic of great interest in the last years. Most applications of WSN require a correlation of the measured value with the location of the measurement. Quite some research is being undergone recently on localization methods $[1,20,21]$. Among the best established methods is the use of RSS values of radio frequency (RF) signals for localization. Due to the simplicity of RSS localization and the availability on most sensor nodes, this method has been carefully examined $[2,11,14,24]$. In spite of the amount of published work on the topic, there is still a lack of application-specific real-world evaluations of localization approaches. The following brief overview of the state of the art is focused on practically evaluated 
RSS localization approaches on the one hand and applications of Bayesian estimators for position estimation and tracking on the other.

\subsection{RSS Localization in WSN}

The approaches that make use of signal strength information for localization in WSN can mostly be classified into range-based and range-free methods [16, 20]. One example for range-free RSS localization are so-called fingerprinting techniques that record RSS values of different neighboring nodes. The position can then be estimated by finding the best match to a previously recorded data base. Different kinds of approaches in this direction have been presented $[2,11,12]$. Alternatively, RSS measurements allow to estimate the distance between a sender and a receiver. For this, transmit power, antenna characteristics and propagation path have to be known to some extend [23,24]. The calculated distances can be used to estimate the position of the node by means of geometric approaches like trilateration or other methods. In [24] the authors present experimental results for a range based localization system in an outdoor test area. In [16] the authors combine fingerprinting with RSS-distance measurements and come to the conclusion that this allows to improve localization accuracy in spite of the large fluctuations of the RSS values.

However, a lot of the published experimental evaluations lack real-world applicability. Mostly, a line of sight (LoS) connection is assumed and, if indoor scenarios are considered at all, the experimental evaluations are limited to a few rooms. The large fluctuations of RSS values due to multi-path fading and other effects makes accurate localization difficult, especially in static networks.

\subsection{Bayesian State Estimation for WSN Localization}

To cope with these fluctuations, Bayesian state estimators such as the Kalman filter, Particle filters or others have been used for position estimation and tracking of robots or persons for some time [5]. In [6], the authors present a Kalman filter indoor tracking system based on WiFi measurements of mobile phones. The system is calibrated for the use in a specific environment and permits to localize the devices. Using a version of the UKF, the authors of [18] present a moving person tracking system based upon an RSS map. A concept to localizing the anchor nodes is presented in [17], where the authors propose to use a mobile robot to collect signal strength data and a Kalman filter to cope with the high variances. Other Bayesian approaches like particle filters are used to estimate the location of nodes in a sensor network in various works. In [14], the authors present a method to localizing and tracking a mobile node based on RSS measurements.

However, not much work has been done on practical evaluations of Kalman filters for person tracking in WSN. Especially the application of these approaches on real-world data and the actual implementation and evaluation of a larger scale ad-hoc localization WSN has not yet been sufficiently evaluated. 


\section{Person Localization System Model and RSS Measurement Model}

In this paper, the dynamic localization of a person in 2-dimensional space is considered. The state vector $\underline{x}_{k}=\left[\underline{x}_{k, P}, \underline{x}_{k, V}\right]^{\mathrm{T}}$ thus consists of the persons' position $\underline{x}_{k, P} \in \mathbb{R}^{2}$ and velocity $\underline{x}_{k, V} \in \mathbb{R}^{2}$. In these terms, $k=0,1, \ldots$ represents the discrete time index.

\subsection{System Model}

The moving person can thus be described by means of a linear discrete-time dynamic system.

$$
\underline{x}_{k+1}=\mathbf{A} \cdot \underline{x}_{k}+\underline{\boldsymbol{w}}_{k}
$$

Herein $\underline{\boldsymbol{w}}_{k} \sim \mathcal{N}\left(\underline{0}, \mathbf{C}_{k}^{w}\right)$ is a zero-mean Gaussian noise term with covariance matrix $\overline{\mathbf{C}}_{k}^{w}$. For the considered position velocity model, the system matrix $\mathbf{A}$ and the process covariance $\mathbf{C}^{\omega}$ are defined by:

$$
\mathbf{A}=\left[\begin{array}{cc}
\mathbf{I} & T \cdot \mathbf{I} \\
\mathbf{0} & \mathbf{I}
\end{array}\right], \mathbf{C}^{\omega}=\left[\begin{array}{cc}
\frac{T^{3}}{3} \mathbf{C}_{c}^{\omega} & \frac{T^{2}}{2} \mathbf{C}_{c}^{\omega} \\
\frac{T^{2}}{2} \mathbf{C}_{c}^{\omega} & T \mathbf{C}_{c}^{\omega}
\end{array}\right]
$$

where $T$ is the sampling time, $\mathbf{I}$ the identity matrix and $\mathbf{C}_{c}^{\omega}=\operatorname{diag}\left(\left[C_{c, x}^{\omega}, C_{c, y}^{\omega}\right]\right)$ the covariance matrix of the process noise from the continuous-time system model.

\subsection{Measurement Model}

In this system, an RSS distance measurement $\hat{y}_{k}^{(i)}$ at time step $k$ represents the Euclidean distance

$$
h^{(i)}(\underline{x})=\left\|\underline{x}_{k, P}-\underline{l}^{(i)}\right\|_{2}
$$

of the moving person at position $\underline{x}_{k, P}$ to the $i$-th anchor node at position $\underline{l}^{(i)}$. The measurement equation

$$
\hat{y}_{k}^{(i)}=h^{(i)}\left(\underline{x}_{k}\right)+\boldsymbol{v}_{k}^{(i)}
$$

is thus non-linear with the zero-mean Gaussian noise term $\boldsymbol{v}_{k}^{(i)} \sim \mathcal{N}\left(0, c_{k}^{(i)}\right)$ with variance $c_{k}^{(i)}$. For the RSS range-measurements of the distances the log-distance path-loss model

$$
P L=P+10 n \cdot \log _{10}\left(\frac{d}{d_{0}}\right)+N_{G}
$$

is used [19]. This model results from the Friis' free-space equation and is commonly used as a simple approximation of the signal strength drop-off over the distance. The path-loss $P L[d B m]$ for a given distance $d$ is expressed as a function 
of the path-loss coefficient $n$, a reference measurement $P$ of the received power at distance $d_{0}$, and a normally distributed noise term $N_{G}$. If $d_{0}$ is assumed to be $1 \mathrm{~m}$, the parameters $P$ and $n$ can be determined experimentally. The inversion of this function can then be used to estimate the distance $\hat{y}^{(i)}(P L)$ between a sender and a receiver for a given RSS measurement on given hardware.

\section{Considered Localization Approaches}

The optimal approach toward estimating the uncertain state $\underline{\boldsymbol{x}}_{k}$ of the system, i.e., position and velocity of the person, is to calculate its probability density function $f\left(\underline{x}_{k}\right)$ by means of the system and measurement model (1) and (4), respectively. Although only normally distributed perturbations $\underline{\boldsymbol{w}}_{k}$ and $\underline{\boldsymbol{v}}_{k}$ are assumed, the density of the state will then be non-Gaussian due to the nonlinearity (4) and can even be multi-modal. Thus, in order to derive tractable estimation techniques, approximate solutions are inevitable. Nonlinear estimators, such as particle filters or Gaussian sum filters, are computationally very demanding and are impractical on a WSN-typical low-power microcontroller unit (MCU). Therefore, the use of Kalman filter derivatives is considered in this paper. These filters preserve the Gaussianity of the state estimate and hence allow for a simple parameterization of the state estimate by the corresponding mean $\underline{\hat{x}}_{k}$ and covariance matrix $\mathbf{C}_{k}$. The considered Kalman filter techniques are compared to a closed-form solution, i.e., a static localization approach that utilizes no motion model.

\subsection{Closed-Form Solution (CFS)}

The closed-form solution [7] directly computes an estimate for the position $\underline{\boldsymbol{x}}_{k, P}$ by means of a least-squares fitting of the observations. It does not make use of a motion model, such as (1). Therefore, the velocity $\underline{\boldsymbol{x}}_{k, V}$ can be omitted. This solution employs a squared and expanded formulation

$$
\left(\hat{y}_{k}^{(i)}\right)^{2}=\left\|\underline{x}_{k, P}\right\|_{2}^{2}-2\left(\underline{l}^{(i)}\right)^{\mathrm{T}} \cdot \underline{x}_{k, P}+\left\|\underline{l}^{(i)}\right\|_{2}^{2}
$$

of the measurement equation (3). For $N$ available measurements at a time instant $k$, the measurement equations can be reformulated and summarized as

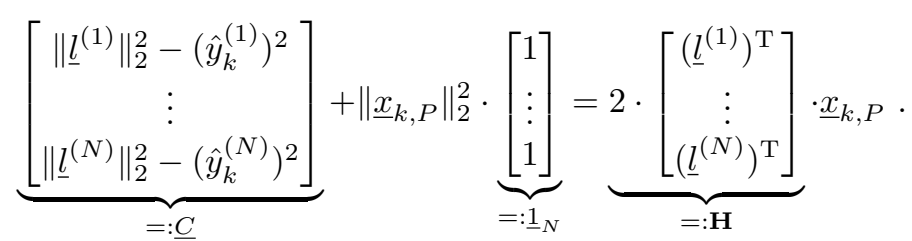

As described in $[7,22]$, a least-squares solution is given by

$$
\underline{\hat{x}}_{k, P}=\mathbf{G} \cdot \underline{C}+\left\|\underline{x}_{k, P}\right\|_{2}^{2} \cdot \mathbf{G} \cdot \underline{1}_{N}
$$


with $\mathbf{G}:=\left(\mathbf{H}^{\mathrm{T}} \mathbf{C}_{k}^{v} \mathbf{H}\right)^{-1} \mathbf{H}^{\mathrm{T}} \mathbf{C}_{k}^{v}$, where $\mathbf{C}_{k}^{v}=\operatorname{diag}\left[\mathrm{c}_{\mathrm{k}}^{(1)}, \ldots \mathrm{c}_{\mathrm{k}}^{(\mathrm{N})}\right]$ comprises the individual sensor variances. In order to eliminate $\left\|\underline{x}_{k, P}\right\|_{2}^{2}$ in (7), squaring (7) provides

$$
\left(\mathbf{G} \underline{1}_{N}\right)^{\mathrm{T}}\left(\mathbf{G} \underline{1}_{N}\right)\left\|\underline{x}_{k, P}\right\|_{2}^{4}+\left(2(\mathbf{G} \underline{C})^{\mathrm{T}} \mathbf{G} \underline{1}_{N}-1\right)\left\|\underline{x}_{k, P}\right\|_{2}^{2}+(\mathbf{G} \underline{C})^{\mathrm{T}}(\mathbf{G} \underline{C})=0,
$$

which can be converted according to $\left\|\underline{x}_{k, P}\right\|_{2}^{2}$ and inserted into (7), which yields an estimate $\underline{\hat{x}}_{k, P}$. The closed-form approach is only applicable if (6) is not underdetermined, so that (7) can be solved. This condition is fulfilled if at least three measurements are available.

The closed-form solution is strongly related to a maximum-likelihood estimation of the position [22]. This has to be considered as a weak spot of this approach, since no prior information, i.e., knowledge about previous positions, is incorporated. Compared with this, a dynamic localization approach uses information about the movement between two measurement steps, is therefore less susceptible to measurement errors and can provide smoother trajectories.

\subsection{Extended Kalman Filter (EKF)}

The Kalman filter [10] formulas provide an optimal solution for linear systems and measurement models corrupted by additive Gaussian noise, where the stochastic state estimate can then uniquely be parameterized by the conditional mean $\underline{\hat{x}}_{k}$ and covariance matrix $\mathbf{C}_{k}$. In order to apply the Kalman filter to the nonlinear sensor equation (4), it appears to be most apparent to linearize the model by a first-order Taylor series expansion

$$
h^{(i)}\left(\underline{x}_{k}\right) \approx h^{(i)}\left(\underline{\hat{x}}_{k}^{\mathrm{p}}\right)+\underbrace{\left(\left.\frac{\partial h^{(i)}}{\partial \underline{x}_{k}}\right|_{\underline{x}_{k}=\underline{\hat{x}}_{k}^{\mathrm{p}}}\right)}_{=: \mathbf{H}_{k}^{(i)}}\left(\underline{x}_{k}-\underline{\hat{x}}_{k}^{\mathrm{p}}\right),
$$

where $h^{(i)}$ is approximated at the current state estimate $\underline{\hat{x}}_{k}^{\mathrm{p}}=\left(\left[\underline{x}_{k, P}, \underline{x}_{k, V}\right]^{\mathrm{T}}\right)^{\mathrm{p}}$. For an observation $\hat{y}_{k}^{(i)}$, the Kalman fusion step then becomes

$$
\underline{\hat{x}}_{k}^{\mathrm{e}}=\underline{\hat{x}}_{k}^{\mathrm{p}}+\mathbf{K}_{k}\left(\hat{y}_{k}^{(i)}-h^{(i)}\left(\underline{\hat{x}}_{k}^{\mathrm{p}}\right)\right)
$$

and

$$
\mathbf{C}_{k}^{\mathrm{e}}=\mathbf{C}_{k}^{\mathrm{p}}-\mathbf{K}_{k} \mathbf{H}_{k}^{(i)} \mathbf{C}_{k}^{\mathrm{p}}
$$

for the estimated mean $\underline{\hat{x}}_{k}^{\mathrm{e}}$ and covariance matrix $\mathbf{C}_{k}^{\mathrm{e}}$, respectively. The matrix $\mathbf{K}_{k}$ denotes the Kalman gain

$$
\mathbf{K}_{k}=\mathbf{C}_{k}^{\mathrm{p}}\left(\mathbf{H}_{k}^{(i)}\right)^{\mathrm{T}}\left(c_{k}^{(i)}+\mathbf{H}_{k}^{(i)} \mathbf{C}_{k}^{\mathrm{p}}\left(\mathbf{H}_{k}^{(i)}\right)^{\mathrm{T}}\right)^{-1} .
$$

The predicted mean $\underline{\hat{x}}_{k}^{\mathrm{p}}$ and predicted covariance matrix $\mathbf{C}_{k}^{\mathrm{p}}$ are obtained by means of the motion model (1) from

$$
\underline{\hat{x}}_{k}^{\mathrm{p}}=\mathbf{A} \underline{\hat{x}}_{k-1}^{\mathrm{e}}
$$


and

$$
\mathbf{C}_{k}^{\mathrm{p}}=\mathbf{A C}_{k-1}^{\mathrm{e}} \mathbf{A}^{\mathrm{T}}+\mathbf{C}_{k}^{w},
$$

respectively. In order to reduce the computational complexity of the fusion step, when multiple measurements have to be dealt with at once, an inverse covariance formulation of the Kalman filter, the information filter [15], can be used. The formulas of the state prediction are then more involved.

By employing the state prediction as prior knowledge in each fusion step, the estimation results can be improved significantly. Unfortunately, the EKF can severely be affected by linearization errors, which often results in an underestimated covariance matrix (9).

\subsection{Unscented Kalman Filter (UKF)}

In order to overcome the drawbacks of the EKF, the unscented Kalman filter [9] linearizes the measurement function by means of a linear regression analysis. For that purpose, a set of $L$ regression points $\mathcal{X}_{i}$ around the prior state estimate $\underline{\hat{x}}_{k}^{\mathrm{p}}$ is chosen, for which the corresponding function values

$$
\mathcal{Y}_{i}=h^{(i)}\left(\mathcal{X}_{i}\right)
$$

are calculated. The linearization $h^{(i)}\left(\underline{x}_{k}\right) \approx \mathbf{H}^{(i)} \underline{x}_{k}+\underline{d}$ is then obtained from the least-squares fit

$$
\{\mathbf{H}, \underline{d}\}=\arg \min _{\mathbf{H}, \underline{d}} \sum_{i=1}^{L} \omega_{i} \cdot \underline{e}_{i}^{\mathrm{T}} \cdot \underline{e}_{i},
$$

where the weighted sum of squared errors $\underline{e}_{i}=\mathcal{Y}_{i}-\left(\mathbf{H} \mathcal{X}_{i}+\underline{d}\right)$ is minimized. This least-squares problem is solved by

$$
\mathbf{H}=\mathbf{C}_{x y}^{\mathrm{T}} \mathbf{C}_{x x}^{-1} \text { and } \underline{d}=\overline{\mathcal{Y}}-\mathbf{H} \cdot \overline{\mathcal{X}}
$$

with $\overline{\mathcal{X}}=\sum_{i=1}^{L} \omega_{i} \cdot \mathcal{X}_{i}, \overline{\mathcal{Y}}=\sum_{i=1}^{L} \omega_{i} \cdot \mathcal{Y}_{i}, \mathbf{C}_{x x}=\sum_{i=1}^{L} \omega_{i} \cdot\left(\mathcal{X}_{i}-\overline{\mathcal{X}}\right) \cdot\left(\mathcal{X}_{i}-\overline{\mathcal{X}}\right)^{\mathrm{T}}$, and $\mathbf{C}_{x y}=\sum_{i=1}^{L} \omega_{i} \cdot\left(\mathcal{X}_{i}-\overline{\mathcal{X}}\right) \cdot\left(\mathcal{Y}_{i}-\overline{\mathcal{Y}}\right)^{\mathrm{T}}$. Note that $\overline{\mathcal{X}}$ is equal to $\underline{\hat{x}}_{k}^{\mathrm{p}}$ for symmetrically chosen regression points. Subsequently, this linearized mapping can be used to calculate the estimated mean (8) and covariance matrix (9). The UKF renders, in general, a significantly better estimation quality than the EKF. Of course, the quality depends on the number and placement of the regression points and the estimation may fail if the regression points do not capture the nonlinearity properly.

\subsection{Analytic Moment Calculation (AMC)}

The linear regression analysis can, in many situations, be replaced by an analytic moment calculation, as described in [3]. The mean $\overline{\mathcal{Y}}$ and the covariance matrices 
$\mathbf{C}_{x y}$ and $\mathbf{C}_{x x}$ for (10) can then be calculated in closed-form. More precisely, the underlying integrals for these moments are directly computed. Of course, the integrals cannot, in general, be solved efficiently and without numerical integration, but in the considered localization scenario, this concept is applicable, if a noise before nonlinearity observation model

$$
\hat{y}_{k}^{(i)}=\tilde{h}^{(i)}\left(\underline{x}_{k}, \boldsymbol{v}_{k}^{(i)}\right):=\left(h^{(i)}\left(\underline{x}_{k}+\operatorname{diag}\left[\boldsymbol{v}_{k}^{(i)}, \boldsymbol{v}_{k}^{(i)}\right]\right)\right)^{2},
$$

is used instead of the model (4). Then, the integrals become simple matrix-vector operations [3]. The AMC provides an optimal stochastical linearization of the nonlinear measurement mapping and thus promises the best estimation results.

\section{Experimental Evaluation}

To evaluate these methods, experimental data were collected by means of a 65-node WSN testbed. The used LocNode sensor nodes consist of a Texas Instruments MSP430 low-power MCU and an IEEE 802.15.4 compliant 2.4 GHz transceiver CC2520. One version (simple LocNode) is designed to fit in a robust $5.5 \times 2 \times 2.5 \mathrm{~cm}^{3}$ casing, whereas the other (extended LocNode) allows for the connection of an SD-card holder or a GPS module via two 20-pin expansion board connectors. Both versions incorporate a PCB antenna and an identical RF design (Fig. 1).
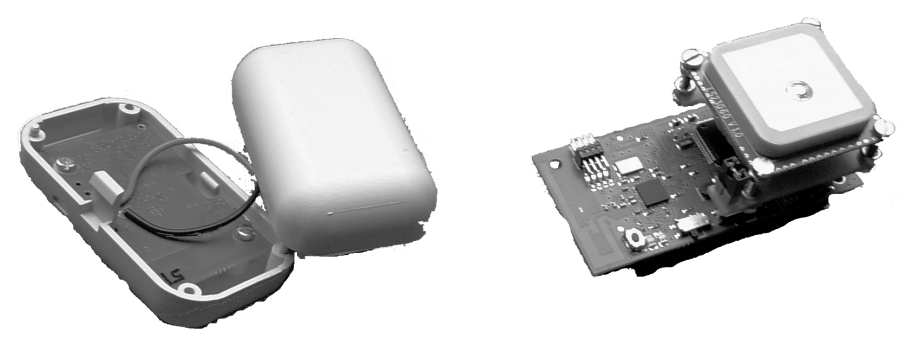

Fig. 1. LocNode: simple version in casing and extended version with attached GPS module.

\subsection{Setup}

60 nodes were deployed in an out- (football field) and indoor (one floor, office building) setting (Fig. 2). Outdoors, the nodes were placed on the ground in the grass due to a lack of adequate stands, indoors, the nodes were placed on tables, window ledges or name plates at the office doors at a height between $1 \mathrm{~m}$ and 1.5 $m$. These anchor nodes were set to broadcast their positions at a per-node rate of $4 \mathrm{~Hz}$. To allow for real-world evaluation of the considered person localization, 

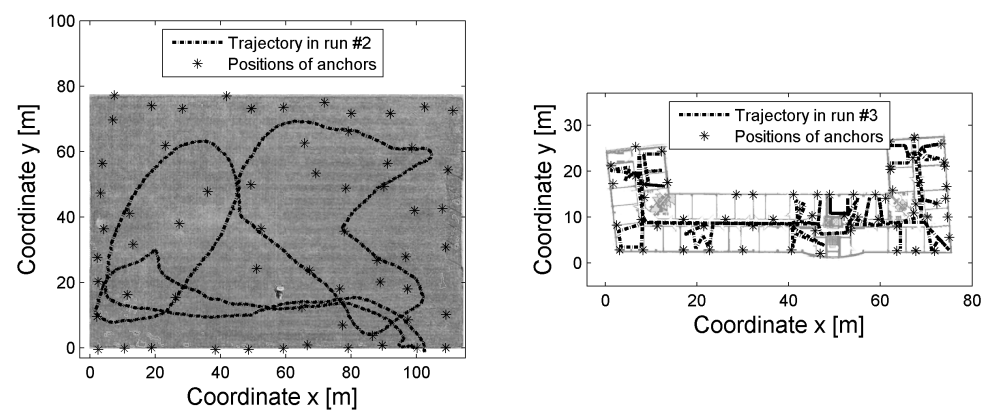

Fig. 2. Positions of anchor nodes and exemplary ground truth trajectories for out(left) and indoor (right) experimental setup.

the data collection was carried out as application-realistic as possible. To be able to analyze varying positions of the node carried by the user, five mobile nodes (on-body nodes) were used for the data collection. These on-body nodes were carried on different spots on the test person's body. Two nodes were hanging on lanyards around the test persons' neck in front of the chest and behind the back, one was mounted on a rucksack structure to provide line of sight connection in all directions and two were carried in the right and left trouser pockets. For the off-line data analysis, the packets from all anchor nodes within range were stored on an SD-card on each on-body node. The corresponding real position, i.e., the ground truth, is established with an inertially assisted GPS outdoors and a time based predefined trajectory recording indoors (way point markers on the floor, synchronization with a stop watch). 5 runs were conducted indoors, 9 runs were conducted outdoors. The test persons walking speed was usually varying between 0.5 and $1.5 \mathrm{~m} / \mathrm{s}$ to allow for a realistic movement pattern.

\subsection{Data Collection}

Each of the two experiments lasted approximately two hours and a total of about 2, 000, 000 packets were collected with the five on-body nodes in 9 respectively 5 runs of 6 to 18 minutes. Fig. 3 shows a representation of the collected data for the in- and outdoor experiments. As expected, the path-loss in outdoor environments is smaller than indoors. The coefficients $P$ and $n$ represent an optimal leastsquares fit of the log-distance path-loss model (5) to the cumulated data from all nodes in all experiments. It can be seen that the standard deviation of the estimated distance can be approximated with $\sigma=\frac{d}{2}$ as a function of the distance. This is used as measurement noise in the filters. Fig. 4 illustrates the effect of varying settings of $P$ and $n$ on the distance estimation for each recorded RSS value, the effects of varying parameter settings on the localization accuracy are analyzed in the next section. Usually the log-distance model is mainly considered accurate for indoor scenarios. Nonetheless, it can be seen in Fig. 3 that also the outdoor data of the undergone experimental evaluation can be modeled quite 


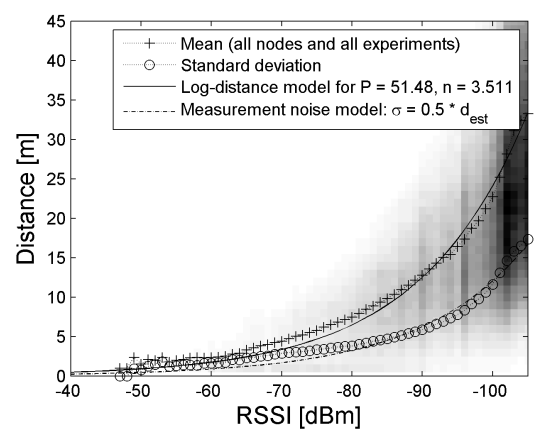

(a) outdoor

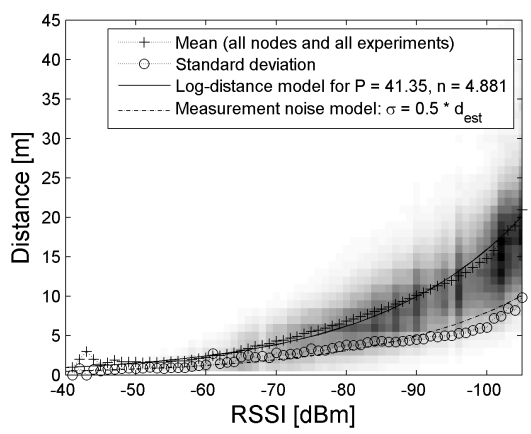

(b) indoor

Fig. 3. Log-distance path-loss model for in- and outdoor scenario (all nodes in all experiments).

accurately. However, it must be pointed out, that this might be an effect of the placement of the anchor nodes on the ground in the wet grass. This results in a substantially lower transmission range. Also, the considered distance is always the Euclidean distance of a projection in the two dimensional plane and the different heights of the on-body nodes are neglected for simplification. Previous experiments with whip-antenna-equipped sensor nodes on tripods at a height of $1 \mathrm{~m}$ above ground showed a different path-loss behavior. Another antenna or RF-design would require an additional calibration. Various other works cope with the behavior of RSS values and path-loss models [13,24,26].

\section{Localization Results and Comparison of Selected Approaches}

Fig. 5 shows an example of reconstructed trajectories with the 4 filters in the outdoor experiment. The dotted line represents the position estimation, the solid line represents the ground truth. The trajectories in this plot are calculated on the data collected by node 3 in the third experimental run. As comparison metric, the root mean square error (RMSE) of the position estimation is used. Fig. 6 shows an equivalent example of an indoor experiment. The trajectories in this plot are calculated on the data collected by node 1 in the third experimental run of each scenario. In the following, the parameters $P$ and $n$ are chosen based on a minimum least-squares fit on the cumulated data collected by all on-body nodes in all runs to prevent over-fitting for a certain on-body node position. The resulting RMSE are calculated with position covariance $\mathbf{C}_{c}^{\omega}=\operatorname{diag}([0.06,0.06])$. The measurement variance $c_{k}=d^{2} / 4$ is chosen based on the experimentally established measurement noise $\sigma=\frac{d}{2}$ (Section 5). Measurements with an RSS under $-99 \mathrm{dBm}$ (receiver sensitivity threshold) are filtered out. Also, a validation gate is used for the Kalman filters to filter out unexpected outliers if the measurement is out of a $95 \%$ confidence interval of the predicted value. The setting of 


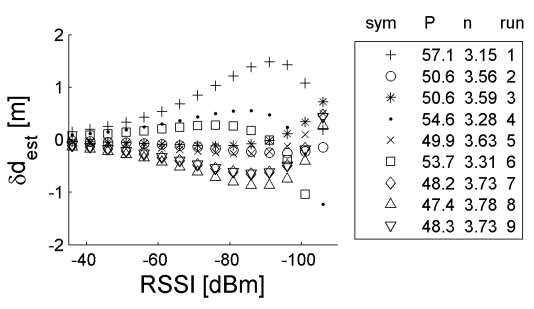

(a) outdoor, different experiments

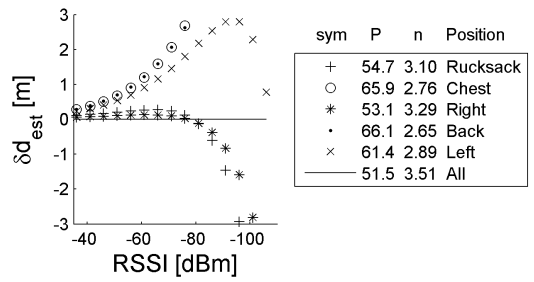

(c) outdoor, different positions

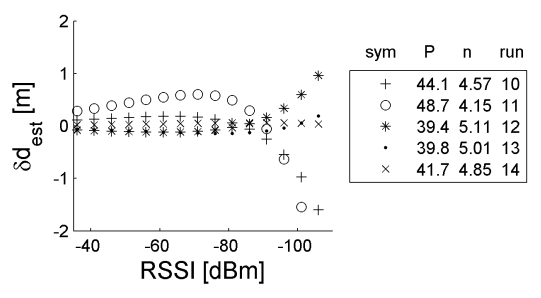

(b) indoor, different experiments

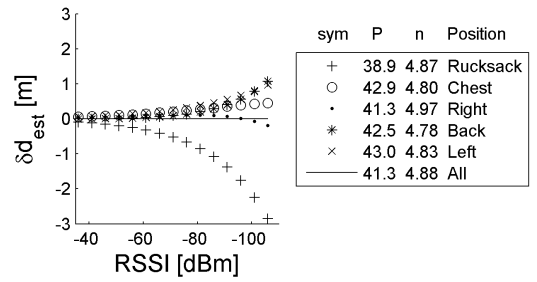

(d) indoor, different positions

Fig. 4. Log-distance path-loss model parameters $P$ and $n$ fitted to different in- and outdoor experiments and different positions of the on-body nodes, effects on distance estimation (difference $\delta d_{\text {est }}$ to distance estimation with $P$ and $n$ as in Fig. 3).

$\mathbf{C}^{\omega}$ would need to be adjusted if the update rate of $4 \mathrm{~Hz}$ was changed. For the collected dataset, a higher $c_{k}$ tends to result in a considerable improvement of the localization accuracy, probably because of the different path-loss behaviors for each on-body node position. For the purpose of this paper, no further optimization of $\mathbf{C}_{c}^{\omega}$ and $c_{k}$ was carried out to prevent over-fitting. Also, no analysis of potential improvements by fusing the data collected by all five on-body nodes is carried out as the intended scenario is to allow the system's user to carry the on-body node at an arbitrarily chosen spot.

Fig. 7 shows a comparison of the resulting root-mean-position-error for the different filters in each test run. The bars represent the resulting errors for the different filters in the different experiments. The mean of all five nodes, as well as the corresponding maximum and minimum for a certain node position is depicted. It can be seen that the compared localization approaches allow for reasonable localization accuracy in all runs. In comparison to the closed form solution that calculates every position without making use of a predicted position and velocity, the inclusion of a system model and a prediction step in the Bayesian information processing approaches improves the results considerably. The chosen parameters allow for reproducible accuracies in all test runs.

Fig. 8 shows a comparison of the different on-body node positions in the inand outdoor experiments. The bars represent the mean of the resulting errors for all in- and outdoor experiments and the maximum and minimum errors of single experiments. All on-body node positions result in a comparable total accuracy in the 9 , respectively the 5 runs. All different positions allow to estimate the trajectory with reasonable accuracy. 

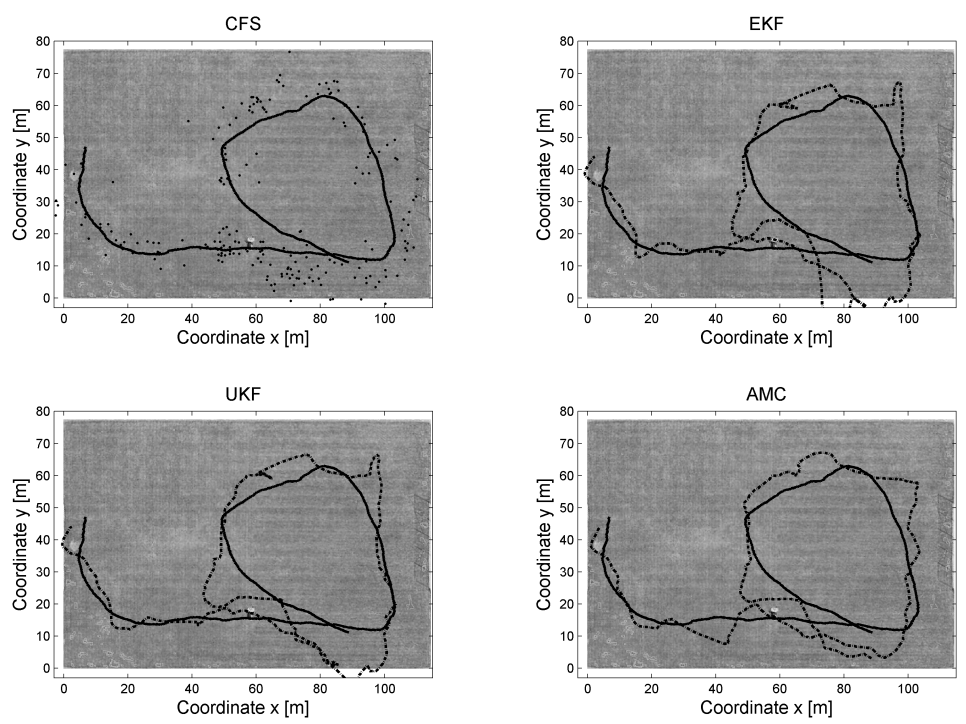

Fig. 5. Estimated trajectories (dotted lines) and corresponding ground truth (solid line) for node 3 in run 3 (outdoor, RMSE: $11.0 m$ (CFS), $9.3 m$ (EKF), $8.8 m$ (UKF), $8.3 m(\mathrm{AMC}))$.

Fig. 9 illustrates the influence of different settings of $P$ and $n$ on the localization accuracy. Run 2 is analyzed exemplary. The parameters are chosen based on least-squares fits of the log-distance model to the collected packets in a single experiment (Run 1-3) or in all in-/outdoor experiments. Again, the bars represent the resulting RMSE of the different on-body node positions. It can be seen that the considered localization methods are very robust against other settings of $P$ and $n$. This results from the comparatively small estimation differences of the different parameter settings in comparison to the measurement variance (Fig. 4). By tuning these parameters for one specific position of the on-body node and/or one specific run, an RMSE of $<1.5 \mathrm{~m}$ is possible.

\subsection{Discussion}

Altogether, the data analysis clearly shows, that for the given scenario it is possible to achieve accuracies on the order of a few meters. As only two data sets are compared, conclusions on the influence of the node density and the actual anchor node positioning cannot be made. Also, a comparison of the accuracies in in- and outdoor environments is difficult because of the difference in the size of the covered area with the same number of nodes. The Bayesian approaches have a clear advantage over the closed-form solution in all evaluations. Although the measured RSS values are subject to strong fluctuations and thus the distance estimations are very noisy, the Bayesian localization approaches provide good position estimations. Additionally, this leads to a robustness against different, re- 

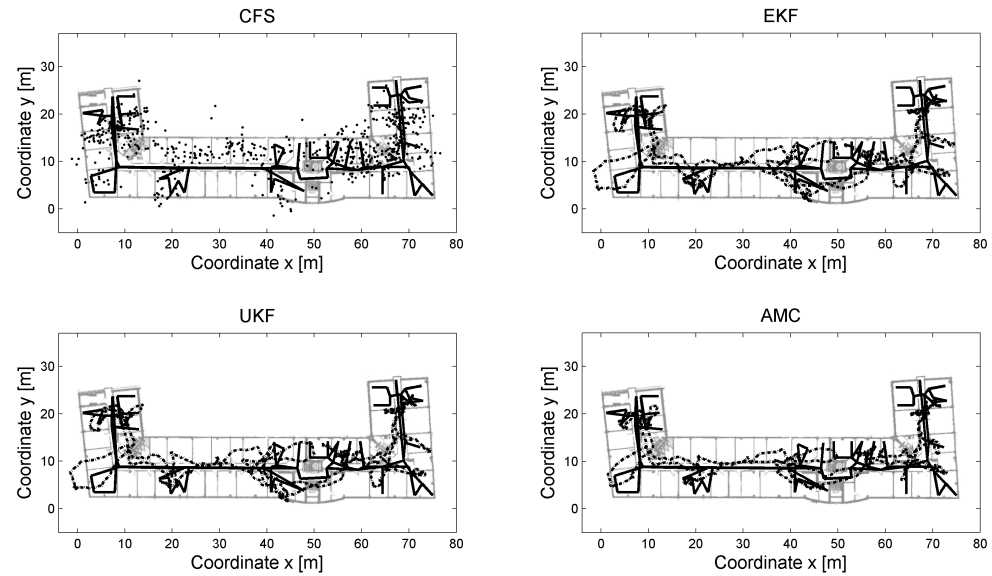

Fig. 6. Estimated trajectories (dotted lines) and corresponding ground truth (solid line) for node 1 in experiment 3 (indoor, RMSE: $5.3 \mathrm{~m}$ (CFS), $3.0 \mathrm{~m}$ (EKF), $3.0 \mathrm{~m}$ (UKF), $2.5 m$ (AMC)).

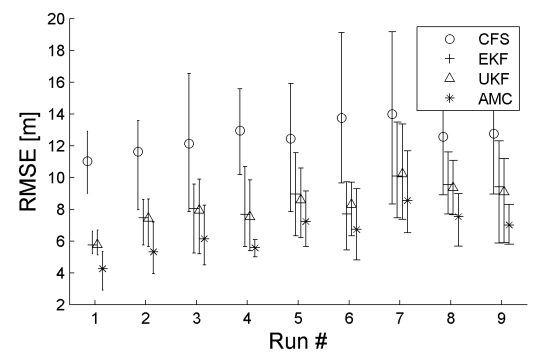

(a) outdoor

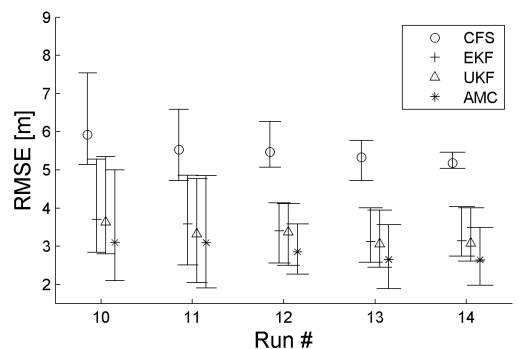

(b) indoor

Fig. 7. RMSE of the different localization approaches for each node position (minimum, maximum and mean for the five node positions).

spectively incorrect path-loss models which allows for little environment-specific calibration. All compared Kalman filter derivatives lead to comparable errors in the evaluated scenario. The AMC and UKF filters perform slightly better, but, due to the good-natured non-linearity of the range measurements, also the EKF provides good position estimates. All three filters can be implemented with a comparable computational effort. As mentioned before, on the established dataset, an increase of the measurement noise results in even smaller localization errors for the UKF and AMC. The EKF however is more sensitive to even slight parameter variations due to the linearization. 


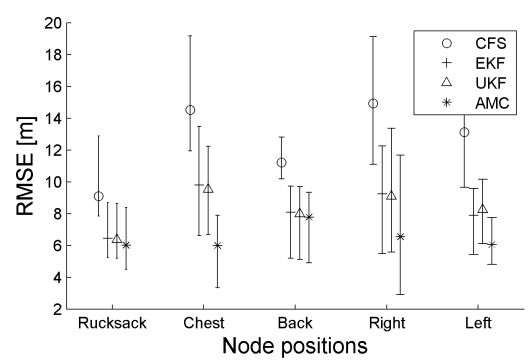

(a) outdoor

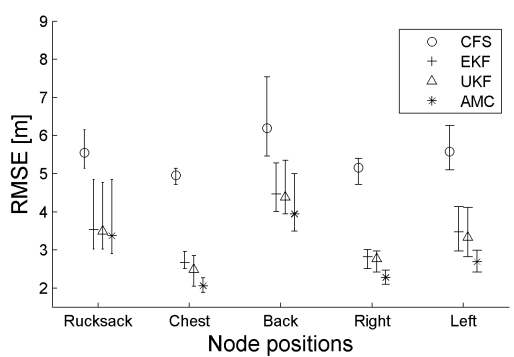

(b) indoor

Fig. 8. RMSE of the different localization approaches for each test run (minimum, maximum and mean for the nine outdoor and five indoor runs respectively).

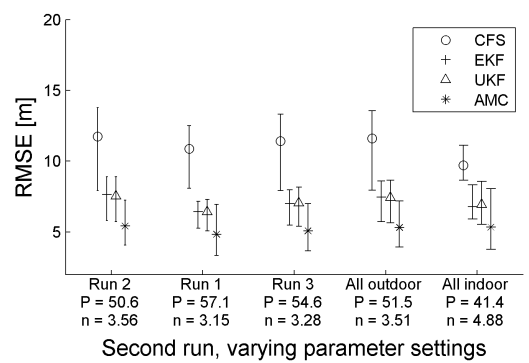

(a) outdoor

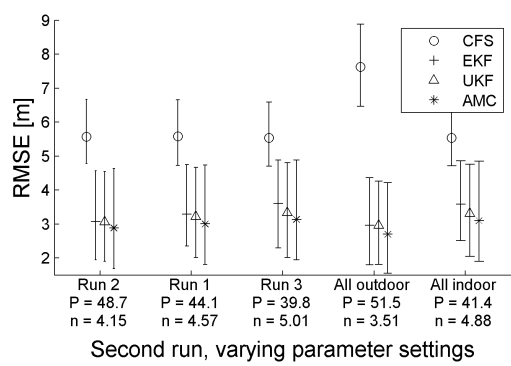

(b) indoor

Fig. 9. Performance of localization approaches for the second run (in- and outdoors) 3 settings.

\section{Conclusion and Future Work}

In this paper, an approach to moving person localization and tracking in a WSN is outlined. Bayesian state estimation is used to fuse successive RSS-range measurements and to estimate the current position and velocity of a person. An experimentally collected data set with a 65-node WSN for both in- and outdoor scenarios is presented and a position velocity system model is established. For the position estimation, three derivatives of the Kalman filter are evaluated and compared with a closed-form solution. In spite of the fluctuations of the RSS measurements and the resulting large errors in the distance estimation, the approaches allow to estimate the position of a moving person in in- and outdoor environments with reasonable accuracy for various applications. It can be concluded, that Bayesian state estimators are a simple, robust and practical method for person localization in WSN. Also, all of the compared methods can principally be implemented on a low-power WSN typical MCU. Thus, a cheap and scalable person localization system can be implemented based on the concepts presented in this paper. 
For the next future, it is planned to further investigate the outlined approaches. The influence of external parameters like node density, weather, experimental surroundings, varying trajectories of moving persons and others is yet to be systematically evaluated. For this, a simulation environment will be designed based on the established behavior.

Additionally, the fusion of the RSS measurements with inertial data from acceleration and gyro sensors is evaluated. With this additional input, it will be possible to refine the movement model and to further improve the resulting localization accuracy. An evaluation of the accuracies when considering an adhoc deployment of the anchor nodes is undergone at the moment. This ad-hoc deployment can e.g. be carried out by means of a pedestrian dead reckoning unit to provide prior position estimates of anchor nodes, which can then be applied to initialize simultaneous localization and mapping algorithms (SLAM). Such a system would enable ad-hoc localization in unknown environments.

\section{Acknowledgment}

This work was supported by the German Research Foundation (DFG) within the Research Training Group GRK 1194 "Self-organizing Sensor-Actuator Networks". The authors would like to thank the DFG for the support.

\section{References}

1. Akyildiz, I., Melodia, T., Chowdhury, K.: A Survey on Wireless Multimedia Sensor Networks. Computer Networks 51(4), 921-960 (2007)

2. Bahl, P., Padmanabhan, V.: RADAR: An In-Building RF-Based User Location and Tracking System. In: IEEE infocom. vol. 2, pp. 775-784 (2000)

3. Beutler, F., Huber, M.F., Hanebeck, U.D.: Optimal Stochastic Linearization for Range-based Localization. In: Proceedings of the 2010 IEEE/RSJ International Conference on Intelligent Robots and Systems (IROS 2010). Taipei, Taiwan (2010)

4. Depenthal, C., Schwendemann, J.: iGPS-a New System for Static and Kinematic Measurements. In: 9th Conference on Optical 3D Measurement Techniques (2009)

5. Fox, D., Hightower, J., Liao, L., Schulz, D., Borriello, G.: Bayesian Filtering for Location Estimation. IEEE Pervasive Computing pp. 24-33 (2003)

6. Guvenc?, I., Abdallah, C., Jordan, R., Dedeoglu, O.: Enhancements to RSS Based Indoor Tracking Systems using Kalman Filters. In: In GSPx \& International Signal Processing Conference (2003)

7. Hanebeck, U.D., Schmidt, G.: Closed-Form Elliptic Location with an Arbitrary Array Topology. In: Proceedings of the IEEE International Conference on Acoustics, Speech, and Signal Processing (ICASSP). pp. 3070-3073. Atlanta, Georgia (1996)

8. Jimenez, A., Seco, F., Prieto, J., Guevara, J.: Indoor Pedestrian Navigation using an INS/EKF Framework for Yaw Drift Reduction and a Foot-mounted IMU. Proceedings of the 7th Workshop on Positioning, Navigation and Communication, March 11-12, 2010, Dresden, Germany (2010) 
9. Julier, S.J., Uhlmann, J.K.: A New Extension of the Kalman Filter to Nonlinear Systems. In: Proceedings of AeroSense: The 11th International Symposium on Aerospace/Defense Sensing, Simulation and Controls. Orlando (1997)

10. Kalman, R.E.: A New Approach to Linear Filtering and Prediction Problems. Transactions of the ASME - Journal of Basic Engineering pp. 35-45 (1960)

11. Lorincz, K., Welsh, M.: MoteTrack: a Robust, Decentralized Approach to RF-based Location Tracking. Personal and Ubiquitous Computing 11(6), 489-503 (2007)

12. Madigan, D., Einahrawy, E., Martin, R., Ju, W., Krishnan, P., Krishnakumar, A.: Bayesian Indoor Positioning Systems. In: Proceedings IEEE INFOCOM 2005. 24th Annual Joint Conference of the IEEE Computer and Communications Societies. pp. $1217-1227(2005)$

13. Mao, G., Anderson, B., Fidan, B.: Path Loss Exponent Estimation for Wireless Sensor Network Localization. Computer Networks 51(10), 2467-2483 (2007)

14. Morelli, C., Nicoli, M., Rampa, V., Spagnolini, U., Alippi, C.: Particle Filters for RSS-based Localization in Wireless Sensor Networks: An Experimental Study. In: Acoustics, Speech and Signal Processing, 2006. ICASSP 2006 Proceedings. 2006 IEEE International Conference on. vol. 4. IEEE (2006)

15. Mutambara, A.G.O.: Decentralized Estimation and Control for Multisensor Systems. CRC Press, Inc., Boca Raton, FL, USA (1998)

16. Noh, A., Lee, W., Ye, J.: Comparison of the Mechanisms of the Zigbee's Indoor Localization Algorithm. In: Ninth ACIS International Conference on Software Engineering, Artificial Intelligence, Networking, and Parallel/Distributed Computing (SNPD'08). pp. 13-18. IEEE (2008)

17. Pathirana, P., Bulusu, N., Savkin, A., Jha, S.: Node Localization using Mobile Robots in Delay-Tolerant Sensor Networks. IEEE Transactions on Mobile Computing pp. 285-296 (2005)

18. Paul, A., Wan, E.: RSSI-Based Indoor Localization and Tracking Using SigmaPoint Kalman Smoothers. Selected Topics in Signal Processing, Journal of IEEE 3(5), 860-873 (2009)

19. Rappaport, T., et al.: Wireless Communications: Principles and Practice. Prentice Hall PTR, Upper Saddle River, NJ (2002)

20. Rudafshani, M., Datta, S.: Localization in Wireless Sensor Networks. In: Proceedings of the 6th International Conference on Information Processing in Sensor Networks. p. 60. ACM (2007)

21. Seco, F., Jiménez, A., Prieto, C., Roa, J., Koutsou, K.: A Survey of Mathematical Methods for Indoor Localization. IEEE International Symposium on Intelligent Signal Processing (WISP) pp. 9-14 (2009)

22. Smith, J., Abel, J.: Closed-Form Least-Squares Source Location Estimation from Range-Difference Measurements. Proceedings of the 1987 IEEE Transactions on Acoustics, Speech and Signal Processing (ICASSP) 35(12), 1661 - 1669 (1987)

23. Sugano, M., Kawazoe, T., Ohta, Y., Murata, M.: Indoor Localization System using RSSI Measurement of Wireless Sensor Network based on Zigbee Standard. In: Proc. IASTED Int. Conf. WSN. pp. 1-6 (2006)

24. Whitehouse, K., Karlof, C., Culler, D.: A Practical Evaluation of Radio Signal Strength for Ranging-Based Localization. ACM SIGMOBILE Mobile Computing and Communications Review 11(1), 52 (2007)

25. Xiao, Z., Hei, Y., Yu, Q., Yi, K.: A Survey on Impulse-Radio UWB Localization. SCIENCE CHINA Information Sciences 53(7), 1322-1335 (2010)

26. Yang, S., Cha, H.: An Empirical Study of Antenna Characteristics toward RFBased Localization for IEEE 802.15.4 Sensor Nodes. Wireless Sensor Networks pp. 309-324 (2007) 